\title{
Flight control system for quad-rotor aircraft based on improved integral separation PID
}

\author{
TIAN Hongpeng ${ }^{1, a}$, LI Lei $^{2, b}$ \\ ${ }^{1}$ School of Xi'an University of Science and Technology, Xi'an Shaanxi, 710054, China \\ ${ }^{2}$ School of Xi'an University of Science and Technology, Xi'an Shaanxi, 710054, China

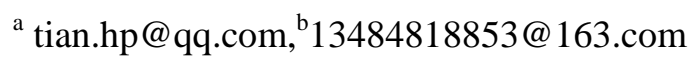

Keywords: quad-rotor aircraft ; PI algorithm; quaternion method; PID control algorithm

\begin{abstract}
Quad-rotor aircraft can be widely used in military and civilian fields, and it is drawing a great deal of attention. Combining with PI algorithm and quaternion method, this article resolves the aircraft flight attitude and complete aircraft attitude control with improved integral separation PID control algorithm. At last, conventional PID controller, integral separation controller and improved integral separation controller are simulated in the environment of MATLAB and compared with each other. The result shows that the response rate of improved integral separation PID is faster than the conventional integral separation algorithm and the overshoot volume is also lower.
\end{abstract}

\section{Introduction}

Because the miniature four rotor aircraft has such prominent features as portable and convenient, simple operation, strong flexibility, vertical ascend or descent, hovering, it has a broad application prospect no matter in the field of military or in the field of civilian ${ }^{[1]}$. However, since the Quad-rotor aircraft is a under actuated system with strong coupling, nonlinear, multivariable, it becomes a key point and difficulty of aircraft research that how to establish a reasonable mathematical model and design control system to make fly stable and safe ${ }^{[2]}$.

The researchers worldwide put forward such control methods as the PID control,the backstepping, and sliding mode control successively ${ }^{[3-5]}$. Among them, the PID algorithm is easy to implement. It also has good stability and high robustness at the same time. But, it still need to be improved in the corresponding speed, overshoot and stability.

\section{Basic flight theory and Modeling}

\subsection{Basic Flight Theory.}

Set the onward direction for the positive direction of the $\mathrm{X}$ axis, and accordingly set the direction of the movement to the left for the positive direction of the $\mathrm{Y}$ axis. Thus, $\mathrm{M}_{1}$ is the motor 1 that falls in the first quadrant, and the rest of the motors are tagging along the clockwise direction in turn. Among them, the $\mathrm{M}_{1}$ and $\mathrm{M}_{3}$ install the anti propeller that rotate along the clockwise direction, and the $\mathrm{M}_{2}$ and $\mathrm{M}_{4}$ install the positive propeller that rotates along the counter clockwise direction. The movement can completed by changing the motor speed to make the quad-rotor aircraft lean some degrees according to table 1 . 
Table 1 Flight status table

\begin{tabular}{|c|c|l|l|l|l|}
\hline $\begin{array}{c}\text { Euler } \\
\text { angle }\end{array}$ & Flight direction & \multicolumn{1}{|c|}{ M1 } & \multicolumn{1}{|c|}{ M2 } & M3 \\
\hline $\begin{array}{c}\text { Pitch } \\
\text { angle } \\
\theta\end{array}$ & forward/ backward & $\begin{array}{l}\text { Equivalent } \\
\text { reduction/ } \\
\text { enlarge }\end{array}$ & $\begin{array}{l}\text { Equivalent } \\
\text { reduction/ } \\
\text { enlarge }\end{array}$ & $\begin{array}{l}\text { Equivalent } \\
\text { enlarge/ } \\
\text { reduction }\end{array}$ & $\begin{array}{l}\text { Equivalent } \\
\text { enlarge/ } \\
\text { reduction }\end{array}$ \\
\hline $\begin{array}{c}\text { Roll } \\
\text { angle }\end{array}$ & to the left/right & $\begin{array}{l}\text { Equivalent } \\
\text { reduction/ } \\
\text { enlarge }\end{array}$ & $\begin{array}{l}\text { Equivalent } \\
\text { enlarge/ } \\
\text { reduction }\end{array}$ & $\begin{array}{l}\text { Equivalent } \\
\text { enlarge/ } \\
\text { reduction }\end{array}$ & $\begin{array}{l}\text { Equivalent } \\
\text { reduction/ } \\
\text { enlarge }\end{array}$ \\
\hline $\begin{array}{c}\text { Yaw } \\
\text { angle } \\
\psi\end{array}$ & turn left/ turn right & $\begin{array}{l}\text { Equivalent } \\
\text { enlarge/ } \\
\text { reduction }\end{array}$ & $\begin{array}{l}\text { Equivalent } \\
\text { reduction/ } \\
\text { enlarge }\end{array}$ & $\begin{array}{l}\text { Equivalent } \\
\text { enlarge/ } \\
\text { reduction }\end{array}$ & $\begin{array}{l}\text { Equivalent } \\
\text { reduction/ } \\
\text { enlarge }\end{array}$ \\
\hline
\end{tabular}

\subsection{Dynamic Model.}

The movement of the quad-rotor aircraft can be seen as translational motion and rotational motion. So we can build a model of these two motions by Newton-Euler equations.

$U_{1}$ is used to express the sun of the body's lift in motion. $U_{2}$ is used to express the rolling torque. $U_{3}$ is used to express the pitching moment. $U_{4}$ is used to express the yaw moment. The rotary inertia of the body around the aircraft coordinate system is expressed by $I_{x}, I_{y}$ and $I_{z}$, and the angular velocity of the shaft is expressed by $\dot{\theta} 、 \dot{\phi} 、 \dot{\psi}$. Then the final dynamic model of the quad-rotor aircraft can be expressed as Formula 1 under the condition of ignoring resistance coefficient in slow flight ${ }^{[6]}$.

$$
\left\{\begin{array}{l}
\ddot{x}=\frac{(\sin \psi \sin \phi+\cos \psi \sin \theta \cos \phi) U_{1}}{m} \\
\ddot{y}=\frac{(\sin \psi \sin \phi \cos \phi-\cos \psi \sin \phi) U_{1}}{m} \\
\ddot{z}=\frac{(\cos \phi \cos \theta) U_{1}-m g}{m} \\
\ddot{\phi}=\frac{\left[U_{2}+\dot{\theta} \dot{\psi}\left(I_{y}-I_{z}\right)\right]}{I_{x}} \\
\ddot{\theta}=\frac{\left[U_{3}+\dot{\phi} \dot{\psi}\left(I_{z}-I_{x}\right)\right]}{I_{y}} \\
\ddot{\psi}=\frac{\left[U_{4}+\dot{\theta} \dot{\phi}\left(I_{x}-I_{y}\right)\right]}{I_{z}}
\end{array}\right.
$$

\section{Controller design}

Since quad-rotor aircraft is a nonlinear complex system of under actuated, strong coupling and multivariable, it is particularly important to design a highly efficient and stable control system to achieve the control of this system. The control logic of the control system is shown in Fig. 1.

\subsection{Attitude Algorithm.}

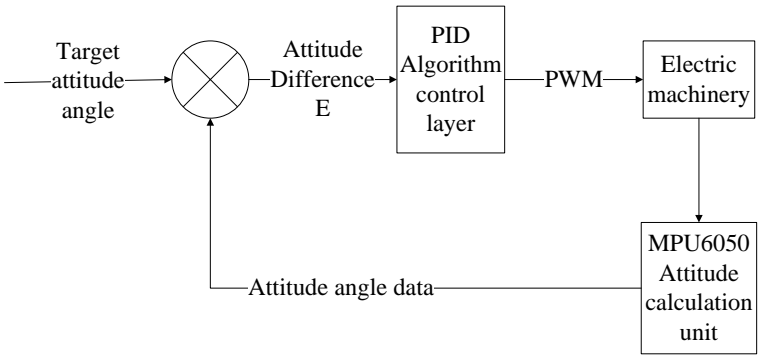

Fig. 1 Control system

The control system of quad-rotor aircraft is divided into two stages of attitude control and attitude control. The first stage is to calculate the attitude of quad-rotor aircraft. Due to the performance of 
the gyroscope in low dynamic state is good but it will appear cumulative error as time goes on, it needs to be corrected. The output of the accelerometer is used to the effect of drift.

PI algorithm to correct angular velocity. At present, the fusion algorithm has Calman filter algorithm, first order complementary filtering algorithm, etc. In this paper, the PI control method is used to eliminate the error of vector product cumulate errors, and then the attitude algorithm is carried out by using the four element method $^{[8]}$.

The error of the angular velocity can be expressed by the vector product of the acceleration and the reference vector as follows:

$$
e=\left(e_{x}, e_{y}, e_{z}\right)
$$

PI control method is a method that only keep the proportion and integral term of the PID control. The cumulative drift of the formula 3 can be used as a correction amount of $\omega$, then the formula 4 is obtained as follows:

$$
e(t)=e(t-1)+K_{i} e(t-1)+K_{P} e(t-1) \quad(3) \quad \omega^{\prime}=\omega+e(t)
$$

In formula 4 , the $\omega$ is the angular velocity. The value is obtained by the gyroscope. $\omega^{\prime}$ is the angular velocity after correction.

Four element attitude calculation. According to the literature[9], the transformation from four elements number converted into Euler angles as follows:

$$
\left\{\begin{array}{l}
\phi=\arctan 2\left(2\left(q_{1} q_{2}+q_{3} q_{4}\right), 1-2\left(q_{2}^{2}+q_{3}^{2}\right)\right) \\
\theta=\arcsin \left(2\left(q_{1} q_{3}+q_{2} q_{4}\right)\right) \\
\psi=\arctan 2\left(2\left(q_{1} q_{4}+q_{2} q_{3}\right), 1-2\left(q_{3}^{2}+q_{4}^{2}\right)\right)
\end{array}\right.
$$

As the body's attitude in a given moment can be expressed with $A=(\theta, \phi, \psi)$, we can get the current attitude of the aircraft.

\subsection{Attitude Control.}

Combine the results of the first stage and the objective attitude to calculate the difference, and then use the PID method to get the output of the motor. Attitude control PID flow chart as shown in Fig. 2:

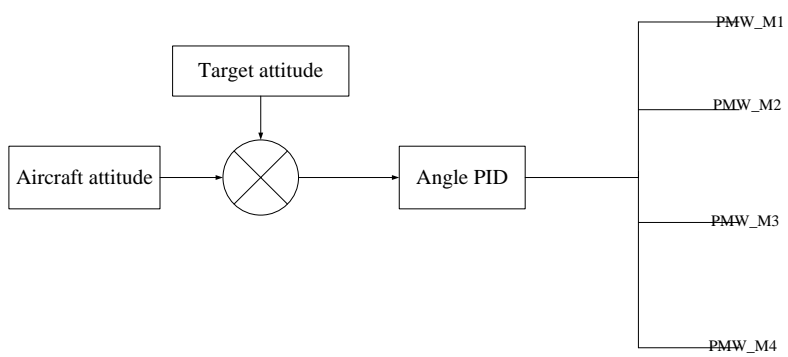

Fig. 2 Attitude control PID flow chart

Based on the integral separation algorithm, an improved algorithm is proposed in this paper. The basic idea is that set two threshold $a$ and $b(a<b)$. When the error is greater than $b$, set the integral control coefficient zero to cancel the integral function; when the error between the two threshold, set the cumulative coefficient zero so that the $e(k)$ no longer accumulated to maintain the original value; when the error is less than $a$, use the complete PID control. Expression as shown in formula 6 .

$$
u(k)=K_{p} e(k)+\beta K_{i}\left\{\sum_{j=0}^{k-1} e(j)+\alpha e(k)\right\} T+K_{d} \frac{(e(k)-e(k-1))}{T}
$$

In this formula, the $\beta$ is the integral term control coefficient whose value is shown in the formula 7. The $\alpha$ is the accumulation term coefficient, and its value is shown as the formula 8 . The $T$ is the sampling period.

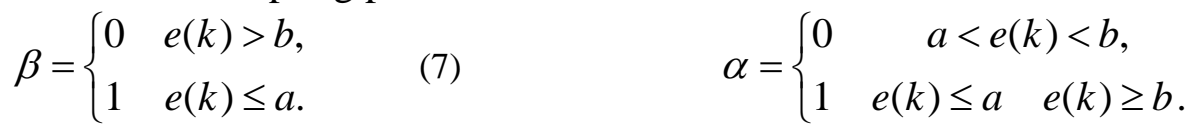


Among them, $\mathrm{a}$ and $\mathrm{b}$ are adjusted according to the test, artificially set the threshold of greater than zero $(\mathrm{a}<\mathrm{b})$.

\section{Simulation analysis}

The conventional PID controller, integral separation controller, and improved integral separation controller are simulated separately in the environment of Matlab,. The results are shown in Fig. 3:

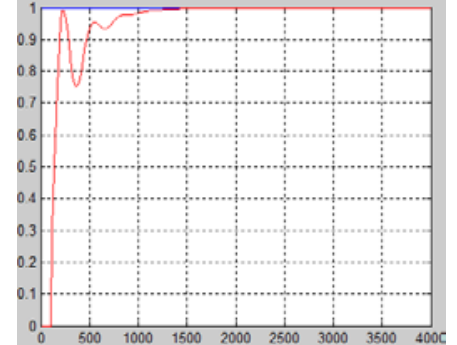

(a) conventional PID control

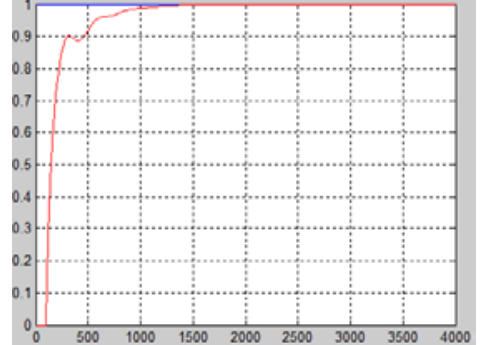

(b) integral separation algorithm (c)improved algorithm

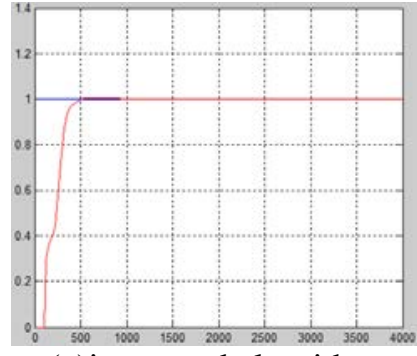

Fig. 3 simulation results

PID parameters are set as that $k_{p}$ is equal to $0.45 ; k_{i}$ is equal to $0.0048 ; k_{d}$ is equal to 12 ; threshold $a$ and $b$ is 0.3 and 0.7 respectively. What can be seen from Fig. 3 is that when using the conventional PID control, the system response time is longer, and the oscillation frequency is more. But when using the integral separation algorithm, the system oscillation frequency is reduced, and the regulating time is shortened. The improved integral separation algorithm has a best effect that almost no oscillations and the shortest adjusting time.

\section{Summary}

Based on an overview of the principle of aircraft flight, this paper uses the PI algorithm combined with the four element method to calculate the flight attitude of the aircraft, and use the improved integral separation PID control algorithm to complete the attitude control of the aircraft. Finally the simulation and comparison of the conventional PID controller, integral separation controller and improved integral separation controller are complete in the environment of MATLAB. The results show that the improved algorithm of integral separation can be overcome the shortcoming of can not eliminate the shortcomings of static caused by not enough integral function. The system has almost no oscillation and adjustment time is shorter than the conventional PID and integral separation PID. Therefore, controller designed by the improved integral separation PID algorithm can make the aircraft fly more stable.

\section{References}

[1] Qi Shuhao. The Design and Attitude Control of A Micro Quadrotor Aircraft [D]. Shanghai Jiao Tong University, 2013.

[2] Xu Yunqing. Research of Quadrotor Unmanned Aerial Vehices on Flying Control[D]. Xiamen University, 2014.

[3] Wu Zhonghua, Jia Qiuling. Several control methods of Quadrotor[J]. Modern Electronic Technology, 2013(15):88-90.

[4] Zhao Yuanwei, Lu Jingzhao. Modeling and Control of a Quadrotor Based on the Backstepping Method[J]. Science technology and Engineering, 2013, 13(34):10425-10430.

[5] Wang Dan. A DMOC-based Research on Trajectory Optimization and Control Algorithms[D]. Beijing Institute of Technology, 2015.

[6] Min B C, Hong J H, Matson E T. Adaptive Robust Control (ARC) for an altitude control of a quadrotor type UAV carrying an unknown payloads[J]. Iccas, 2011, 1416(4):1147-1151.

[7] Yu Yali, Sun Feng, Wang Yuanxi. Hardware circuit design of four-rotor aerial robot based on multi-sensors[J].Transducer and Microsystem,2011, 30(8):113-115. 
[8] Ye Shuqiu. Research of Quard-rotors Aircraft Attitude Control Algorithm [D]. Anhui University Of Science And Technology, 2015.

[9] Jiang Yu, Chen Haiyun, Cen Ruping. Attitude solution algorithm for four rotor aircraft based on four element number [J]. Manufacturing automation, 2015, 37(23):77-80.

[10]Liu Jinkun. Advanced MATLAB control PID simulation (3rd Edition)[M]. Electronic Industry Publishing House, 2011. 